\title{
The weak topology on $q$-convex Banach function spaces
}

\author{
Lucía Agud Albesa
}

Dirigida por: Enrique Sánchez Pérez

Jose Calabuig Rodríguez 


\section{Contents}

1 Motivation. 3

2 Notation and preliminaries. $\quad 4$

2.1 Some properties of Banach lattices and Banach function spaces . . . . . . . . . . . . . 5

2.2 q-convex and q-concave operators, properties. The space $X(\mu)_{[q]} \ldots \ldots \ldots \ldots$

3 A separation argument for Banach function spaces. 12

4 A topological characterization of the q-convexity. 19

5 Applications: A Factorization Theorem for $(q, S)$ convex spaces. 


\section{Motivation.}

Let $1 \leq q<\infty$. The geometric concept of $q$-convexity of Banach lattices and its dual notion - $q$-concavity - are fundamental tools in the study both of the structure of these spaces and the operators defined on them. Although there are earlier results that can be identified with some aspects of the geometric theory of Banach lattices, the main definitions and concepts regarding this subject were introduced in the late sixties and the seventies by Krivine, Maurey and Rosenthal, among others (see [11, 13, 15, 18]). Nowadays, the notion of $q$-convexity in Banach lattices is rather well understood, and also its relation with the main structure theorems of Banach function spaces, $p$-summing operators and factorization of operators through $L^{q}$-spaces (see [13] and also [2, 3, 20] for recent studies on the topic). For instance, it is possible to characterize $q$-convexity in terms of the behavior of positive operators on the space, in particular factorizations through $\ell^{p}$ spaces; see $[3$, Theorem 2.4]. However, no internal characterization of this geometric notion has been given, in the sense that it can be characterized just using elements directly associated with the space (see the section Introduction of [3]). This work has been developed as an attempt of giving a natural and intrinsic characterization of $q$-convexity using topological properties defined by particular classes of seminorms on $X(\mu)$. The following proposition, due to A. Schep, is the starting point of our ideas.

Proposition. ([21, Prop.1.2]) Let $L_{\rho}$ be a p-convex Banach function space with the weak Fatou property. Then there exists a collection $G$ of non-negative measurable functions such that $\rho$ is equivalent to the function norm

$$
\rho_{1}(f)=\sup _{g \in G}\left(\int|f|^{p} g d \mu\right)^{1 / p} .
$$

In this work we analyze in a systematic way the seminorms that appear in the formula above in order to show that the description 
of the weak topology of $q$-convex Banach function spaces is directly related to this family of seminorms.

Another important fact is that $q$-convexity is a property that depends on the lattice, in the sense that, if we give two different norms providing the same topology of a $q$-convex lattice, the $q$ convexity inequality is fulfilled for both of them, although in general with different convexity constants (the constants can be different even in the case that both norms are lattice norms). This fact suggests that this notion must be characterized as a topological property of the lattice. The aim of this work is to show that actually this is the case. After introducing several new weak notions of $q$ convexity, we use them to prove that, if it is possible to define a $q$-concave weak topology on the space and it is a topology between the weak one and the norm topology, then the Banach function space is $q$-convex; the converse is also true. This is done in Section 4. After the introductory Sections 1 and 2, Section 3 is devoted to prove a technical separation theorem that allow us to characterize the weak $q$-convexity notions that are necessary to prove our results. Finally, we present a Maurey-Rosenthal factorization theorem for operators involving weak $q$-convexity and $q$-concavity notions, in order to show how these notions can be used to extend the main arguments that are used in this factorization theory of operators.

\section{Notation and preliminaries.}

The notation that we will use is standard in the theories of Banach spaces and Banach lattices (see [5, 13]). Throughout this work, $(\Omega, \Sigma, \mu)$ will be a $\sigma$-finite complete measure space with $\mu(\Omega)>0$ and $E$ will be a Banach space.

A measure $\mu$ is called $\sigma$-finite if $\Omega$ is the countable union of measurable disjoint sets $\left\{A_{n}\right\}_{n}$ with $\mu\left(A_{n}\right)<\infty, \forall n \in \mathbb{N}$. In these kind of spaces can be built an isometry between them and spaces of finite measure.

We denote by $E^{\prime}$ the topological dual of $E$ and by $B_{E}$ its unit ball. If $S \subseteq X$, we denote by $\operatorname{span}(S)$ the linear covering of the set $S$ in $X$. As usual we write $L(E, F)$ for the space consisting of all bounded linear operators going from the Banach space $E$ into the Banach space $F$ endowed with the operator norm. Given $A \in \Sigma$ the characteristic function $\chi_{A}$ is the function defined by 1 in $A$ and 0 outside of $A$. If $1 \leq q<\infty$ we will write $q^{\prime}$ for the real (extended) 
number in $[1, \infty]$ given by $1 / q+1 / q^{\prime}=1$.

Definition 1 Let $\left(X(\mu),\|\cdot\|_{X(\mu)}\right)$ be a Banach space consisting of (equivalence classes with respect to $\mu$-a.e. equality) locally integrable functions $f: \Omega \rightarrow \mathbb{R}$. We say that $X(\mu)$ is a Banach function space (BFS, or Köthe function space when the space is not necessary complete) when the following conditions hold:

(a) If $f$ is a real measurable function defined on $\Omega$ and $|f| \leq|g|$ for some $g \in X(\mu)$, then $f \in X(\mu)$ and $\|f\|_{X(\mu)} \leq\|g\|_{X(\mu)}$.

(b) $\chi_{A} \in X(\mu)$ for each $A \in \Sigma$ of finite measure.

The second property assures us that the simple functions belong to the space. In vector spaces like $l^{p}$, this property is always satisfied and so, it is omitted in the definition.

\subsection{Some properties of Banach lattices and Ba- nach function spaces}

First of all, we introduce some important definitions for a better comprehension of this work.

Definition 2 Let $(E, \leq,\|\cdot\|)$ be a Banach space. We say that it is a Banach lattice if for all $x, y \in E$ given, $x \wedge y \in E$ and $x \vee y \in E$. Moreover:

$$
\left.\begin{array}{l}
x, y \in E \\
|x| \leq|y|
\end{array}\right\} \Rightarrow\|x\| \leq\|y\|
$$

Definition 3 Let $S$ be a nonempty set of a Banach lattice $E$ that has an upper bound. Then an element $e \in E$ is called the least upper bound (or the supremum, denoted $\sup S$ ) for $S$ if it satisfies the following properties:

1. $e \geq x$ for all $x \in S$.

2. For all $x \in E$, if $x$ is an upper bound for $S$, then $e \leq x$.

Definition $4 A$ directed set is a partially ordered set $(A, \leq)$ such that whenever $a, b \in A$ there is an $x \in A$ such that $a \leq x$ and $b \leq x$.

$A$ directed set is sometimes called an upward-directed set. We may also define the dual notion: a downward-directed set (or filtered set) is a partially ordered set $(A, \leq)$ such that whenever $a, b \in A$ there is an $x \in A$ such that $x \leq a$ and $x \leq b$. 
Definition 5 A real vector space $E$ which is ordered by some order relation $\leq$ is called a vector lattice if any two elements $x, y \in E$ have a least upper bound denoted by $x \vee y=\sup (x, y)$ and a greatest lower bound denoted by $x \wedge y=\inf (x, y)$ and the following properties are satisfied:

1. $x \leq y$ implies $x+z \leq y+z$ for all $x, y, z \in E$,

2. $0 \leq x$ implies $0 \leq t x$ for all $x \in E$ and $t \in \mathbb{R}^{+}$.

Definition 6 A norm $\|\cdot\|$ on a vector lattice $E$ is called a lattice norm if:

$$
|x| \leq|y| \Rightarrow\|x\| \leq\|y\|, \text { for all } x, y \in E
$$

We can conclude that a Banach lattice is a real Banach space E endowed with an ordering $\leq \operatorname{such}$ that $(E, \leq)$ is a vector lattice and the norm on $\mathrm{E}$ is a lattice norm, (see Definition 2).

Definition 7 Let $X$ a Banach lattice is called order complete if all ordered bounded set admits l.u.b. (least upper bound). And it be called order continuous if for all downward directed set, $\left\{x_{\alpha}\right\}_{\alpha \in A}$ such that $\wedge_{\alpha \in A} x_{\alpha}=0$, then $\lim _{\alpha}\left\|x_{\alpha}\right\|=0$.

The same definitions can be given for $\sigma$-order complete and $\sigma$ order continuous if we work with sequences instead ordered sets.

Proposition 1 Let $X$ a Banach lattice, then the following sentences are equivalents:

- $X$ is $\sigma$-order complete and $\sigma$-order continuous.

- All increasing ordered bounded sequences in $X$ are convergent.

- $X$ is $\sigma$-order continuous

- $X$ is order continuous and order complete.

Proposition 2 Let $X(\mu)$ Banach function space, it is satisfied:

1. $X(\mu)$ is a Banach lattice (the order is ' $\leq$ ' $\mu$-a.e.),

2. $X(\mu)$ is $\sigma$-order complete. 
Another important property in these spaces is that in the Banach function spaces, each convergent sequence admits a $\mu$-a.e. convergent subsequence.

Definition 8 Let $L^{0}(\mu)$ be the space of equivalence classes of $\mu$ almost everywhere equal measurable functions.

Definition 9 The Köthe dual of $X(\mu)$, that is, $X(\mu)^{\times}$is the vector space of all (classes of $\mu$-a.e. equal) measurable functions $g$ on $\Omega$ such that $f g \in L^{1}(\mu)$ for all $f \in X(\mu)$.

The norm of this space is given by

$$
\|g\|_{X(\mu)^{\times}}:=\sup _{f \in B_{X(\mu)}}\left|\int_{\Omega} f g d \mu\right|, \quad g \in X(\mu)^{\times} .
$$

The Köthe dual is often called the associated space of $X(\mu)$. Given $g \in X(\mu)^{\times}$and let $f \in X(\mu)$, the operator defined by $\phi_{g}: f \longrightarrow$ $\int_{\Omega} f g d \mu$ is continuous. (In the proof of this fact is necessary to use the existence of $\mu$-a.e. convergent subsequence for any convergent sequence).

Definition 10 A Banach function space $X(\mu)$ is said to be $\sigma$-order continuous if every order bounded increasing sequence is convergent. If the space $X(\mu)$ is $\sigma$-order continuous, the set of the simple functions is dense in $X(\mu)$.

Definition $11 X(\mu)$ has the weak Fatou property if for every increasing sequence in $X(\mu), f_{n} \uparrow f$, with sup $\left\|f_{n}\right\|<\infty$ then $f \in$ $X(\mu)$.

It is possible to show the following result, which gives us a characterization of the Köthe dual space.

Proposition 3 (See $[13, p .29]) X(\mu)^{\times}=X(\mu)^{\prime}$ if and only if $X(\mu)$ is $\sigma$-order continuous.

In general, all dual spaces of $\sigma$-order continuous spaces are identified with Banach function spaces. On the other hand, $X(\mu)^{\times}$is an ideal of the dual topological space of $X(\mu)$. For that, $X(\mu)^{\times}$ endowed with the norm induced by the dual topological, is another Banach function space over the same measurable space, (see [16]).

Definition 12 The space $X^{\times}$is called norming of $X^{\prime}$ if $\forall x \in$ $X^{\prime},|| x \|=\sup \left\{\left|\left\langle x, x^{*}\right\rangle\right| ; x^{*} \in B_{X^{\times}}\right\}$. 
Proposition 4 Let $X(\mu)$ be Banach function space. $X(\mu)^{\times}$is norming of $X(\mu)^{\prime}$ iff given $\left\{f_{n}\right\}_{n} \subseteq X, f_{n} \geq 0, f \geq 0$ such that $f_{n} \uparrow f$, then $\left\|f_{n}\right\| \rightarrow\|f\|$.

If the space $X(\mu)^{\times}$is norming of $X(\mu)^{\prime}$, then $X(\mu)$ is isometric to a subspace of $X^{\prime \prime}$. Due to these properties, it can be obtained the Köthe reflexivity or Fatou Property:

Proposition $5 X=X^{\times \times}$iff given $f_{n} \uparrow f$ a.e., $\left\{f_{n}\right\}_{n} \subset X, f_{n} \geq 0$ a.e., $\sup _{n}\left\|f_{n}\right\|<\infty$, then $f \in X$ and $\|f\|=\lim _{n}\left\|f_{n}\right\|$.

So that, since $\left(X(\mu)^{\times},\|\cdot\|_{X(\mu)^{\times}}\right)$is also a Banach function space, for every $f \in X(\mu)$ :

$$
\|f\|_{X(\mu)}=\sup _{g \in B_{X(\mu) \times}}\left|\int_{\Omega} f g d \mu\right| .
$$

In what follows we introduce the definitions of $q$-convexity and $q$-concavity. Let $1 \leq q<\infty$. We refer the reader to [13] for information about these notions.

\section{2 q-convex and q-concave operators, proper- ties. The space $X(\mu)_{[q]}$}

Definition 13 Let $E$ and $F$ be Banach lattices. An operator $T \in$ $L(E, F)$ is said to be $q$-convex if there exists a constant $k \geq 0$ satisfying that for every finite collection $x_{1}, \ldots, x_{n} \in X(\mu)$,

$$
\left\|\left(\sum_{i=1}^{n}\left|T x_{i}\right|^{q}\right)^{\frac{1}{q}}\right\| \leq k\left(\sum_{i=1}^{n}\left\|x_{i}\right\|^{q}\right)^{\frac{1}{q}}
$$

and $q$-concave if there exists a constant $k \geq 0$ such that for every finite collection $x_{1}, \ldots, x_{n} \in X(\mu)$,

$$
\left(\sum_{i=1}^{n}\left\|T x_{i}\right\|^{q}\right)^{\frac{1}{q}} \leq k\left\|\left(\sum_{i=1}^{n}\left|x_{i}\right|^{q}\right)^{\frac{1}{q}}\right\| .
$$

The best constants $k \geq 0$ in the inequalities above are denoted by $\mathbf{M}^{(q)}(T)$ and $\mathbf{M}_{(q)}(T)$ respectively.

Definition 14 A Banach lattice $E$ is said to be q-convex (resp. qconcave) if the identity operator on $E$ is q-convex (resp. q-concave). 
The canonical examples of Banach lattices with these properties are the spaces $L^{q}(\mu)$, that are $q$-convex and $q$-concave for all $1 \leq q \leq \infty$. Also, if $p<r$ then $L^{r}(\mu)$ is $p$-convex, and $L^{r}(\mu)$ is $p$-concave if $r<p$. If a Banach lattice $E$ is $p$-convex and $p$-concave, then it is an abstract $L^{p}$-space and can be identified with a concrete $L^{p}(\mu)$ space (see for instance [12, Ch.6, §17, Th.7] or [11, Cor.2]).

Nowadays, $q$-convexity and $q$-concavity of the usual Banach function spaces are well-known. The interested reader can find this information in [8] for the case of Lorentz spaces, in [6] for Orlicz spaces and in [9] for Calderón-Lozanovskii spaces (see also the references therein).

Definition 15 [22, $\S 15.10]$ Let $X$ be a vector space. A function $\|\cdot\|: X \rightarrow[0, \infty)$ is called a quasi-norm, if

(Q1) $\|x\|=0$ if and only if $x=0$.

(Q2) $\|\alpha x\|=|\alpha| \cdot\|x\|$ for $\alpha \in \mathbb{C}$ and $x \in X$, and

(Q3) there is a constant $K \geq 1$ such that $\|x+y\| \leq K(\|x\|+\|y\|)$ for all $x, y \in X$.

In this case, $X$ is called a quasi-normed space.

It admits a countable base of neighbourhoods of 0 , namely $\{x \in X:\|x\|<1 / n\}$ for $n \in \mathbb{N}$; so $X$ is a metrizable topological vector space. Every quasi-normed space is necessarily locally bounded as a topological vector space, that is, it possesses a bounded neighbourhood of 0, [22, p.159]

The classical examples of these spaces are the Hardy spaces $H^{p}$ for $0<p \leq \infty$ (not normable if $0<p<1$ ), the Lebesgue spaces and the $L^{p}([0,1])$ spaces for $0<p \leq \infty$ (not normable if $0<p<1$ ).

In the following lemma is shown an interesting result about the convexity or concavity of the q-th power spaces of $X(\mu)$, (see [1]):

Lemma 1 Let $0<t<r<\infty$. Then each r-convex quasi Banach function space $X(\mu)$ is t-convex, and

$$
\mathbf{M}^{(t)}(X) \leq \mathbf{M}^{(r)}(X)
$$

In particular, Banach function spaces $X(\mu)$ being 1-convex, are t-convex for each $0<t<1$ 
Ever Banach lattice is 1-convex and $\infty$-concave and the properties 'r-convexity' and 'r-concavity' for $1 \leq r \leq \infty$ are "decreasing and increasing in $\mathrm{r}^{\prime}$, respectively, (see [13]).

The following notion is one of the main tools in the present work.

Definition 16 Given $0<q<\infty$ the $q$-th power $X(\mu)_{[q]}$ of a Banach function space $X(\mu)$ is the set consisting of all (classes of $\mu$-a.e. equal) measurable functions $f$ such that $|f|^{\frac{1}{q}} \in X(\mu)$.

The $q$-th power of a Banach function space is always a linear space of classes of functions of $L^{0}(\mu)$, the space of $\Sigma$-measurable functions. Moreover, the expression

$$
\|f\|_{X(\mu)_{[q]}}=\left\||f|^{\frac{1}{q}}\right\|_{X(\mu)}^{q}, \quad f \in X(\mu)_{[q]},
$$

provides a quasi-norm for $X(\mu)_{[q]}$, so $X(\mu)_{[q]}$ will be a quasi-Banach function space (q-K.f.s. or q-B.f.s.). Actually for $0<q \leq 1$, $\|$. $\|_{X(\mu)_{[q]}}$ is always a norm and $X(\mu)_{[q]}$ is a Banach function space.

Of course, $X(\mu)_{[1]}=X(\mu)$. Even when $X(\mu)$ is a B.f.s., the space $X(\mu)_{[p]}$ may only be a quasi-normed function space (for some $p)$. For instance, if $X(\mu):=L^{1}([0,1])$ and $1<p<\infty$, then $X(\mu)_{[p]}=L^{1 / p}([0,1])$ with $0<1 / p<1$ and so is a non-normable q-B.f.s., whereas for $0<p \leq 1$ we see that $X(\mu)_{[p]}=L^{1 / p}([0,1])$ with $1 \leq 1 / p<\infty$ is actually a B.f.s.

Proposition 6 Let $X(\mu)$ be a q-B.f.s. with quasi-norm $\|\cdot\|_{X(\mu)}$.

(i) Let $0<p \leq 1$. If $\|\cdot\|_{X(\mu)}$ is a norm, then $\|\cdot\|_{X(\mu)_{[p]}}$ is a norm and hence, $\left(X(\mu)_{[p]},\|\cdot\|_{X(\mu)_{[p]}}\right)$ is a B.f.s.

(ii) Let $0<p<\infty$. Then the q-B.f.s. $X(\mu)$ is $p$-convex if and only if its $p$-th power $X(\mu)_{[p]}$ admits a lattice norm equivalent to $\|\cdot\|_{X(\mu)_{[p]}}$. Moreover, it is possible to select an equivalent lattice norm $\eta_{[p]}$ on $X(\mu)_{[p]}$ satisfying

$$
\eta_{[p]}(f) \leq\|f\|_{X(\mu)_{[p]}} \leq\left(\mathbf{M}^{(p)}[X(\mu)]\right)^{p} \cdot \eta_{[p]}(f), \quad f \in X(\mu)_{[p]} .
$$

(iii) Assume that $X(\mu)$ is $p$-convex. Then the lattice quasi-norm $\|\cdot\|_{X(\mu)_{[p]}}$ is a norm if and only if $\mathbf{M}^{(p)}[X(\mu)]=1$. 
(iv) If $X(\mu)$ is $p$-convex for some $1 \leq p<\infty$, then $X(\mu)$ admits a lattice norm equivalent to $\|\cdot\|_{X(\mu)}$.

The proof of the following lemma is routine.

Lemma 2 Let $X(\mu)$ be a q-B.f.s. based on $(\Omega, \Sigma, \mu)$.

(i) For all $0<p, r<\infty$ we have $\left(X(\mu)_{[p]}\right)_{[r]}=X(\mu)_{[p r]}$.

(ii) Given a q-B.f.s. $Y(\mu)$ over $(\Omega, \Sigma, \mu)$, we have $X(\mu) \subseteq Y(\mu)$ if and only if $X(\mu)_{[p]} \subseteq Y(\mu)_{[p]}$ for some/every $p \in(0, \infty)$.

Due to this result, a relevant property in the context of our work regarding $q$-powers is the following: (see. e.g., [2, Lemma 3])

Corollary 1 Let $1<q<\infty$, if the space is $q$-convex then there is an equivalent norm for $X(\mu)_{[q]}$ such that it becomes a Banach function space.

This equivalent norm is defined by, [4]:

$$
\||| f \mid\|_{X(\mu)_{[q]}}:=\inf \left\{\sum_{i=1}^{n}\|f\|_{X(\mu)_{[q]} ;} ;|f| \leq \sum_{i=1}^{n}\left|f_{i}\right|\right\}
$$

Moreover if $X(\mu)$ is $\sigma$-order continuous then $X(\mu)_{[q]}$ is also $\sigma$ order continuous. The reader is referred to $[1,2,3,13,14,17]$ for all the properties and results about $q$-th powers of Banach function spaces.

Let $X(\mu)$ and $Y(\mu)$ be Banach function spaces. We denote by $\mathrm{M}(X(\mu), Y(\mu))$ the set of all multiplication operators, i.e., the set consisting of (classes of $\mu$-a.e. equal) measurable functions $h$ on $\Omega$ for which the multiplication operator $M_{h}: X(\mu) \rightarrow Y(\mu)$ given by $M_{h}(f):=h f$ is defined. This set is a normed space with its natural norm coming from de operator norm of $M_{h}$. One of the interesting properties is that for $1 \leq q<\infty$,

$$
\mathrm{M}\left(X(\mu)_{[q]}, Y(\mu)_{[q]}\right)=\mathrm{M}(X(\mu), Y(\mu))_{[q]},
$$

(see e.g. [14, Section 2]).

Other important properties for the set $\mathrm{M}(X(\mu), Y(\mu))$ where $X(\mu), Y(\mu)$ are even q-BFS, are the following, (see e.g. [17]):

1. $\mathrm{M}(X(\mu), X(\mu))=L^{\infty}(\mu)$, 
2. $\mathrm{M}\left(X(\mu), L^{1}(\mu)\right)=X(\mu)^{\times}$,

3. Given $1 / q+1 / q^{\prime}=1$, then $\mathrm{M}\left(L^{q}(\mu), L^{1}(\mu)\right)=L^{q}(\mu)^{\prime}=$ $L^{q^{\prime}}(\mu)$

\section{A separation argument for Banach func- tion spaces.}

Let $X(\mu)$ and $Y(\mu)$ be Banach function spaces and let $1 \leq q<\infty$. We start with a technical separation theorem that will allow us to prove the results of this section. The argument is based on the following fundamental tool of the convex analysis.

Lemma 3 (Ky Fan Lemma [5, p.190]) Let $W$ be a compact convex subset of a Hausdorff topological vector space and let $\Psi$ be a concave collection of lower semi-continuous, convex, real functions on $W$. Let $c \in \mathbb{R}$. Suppose, for every $\psi \in \Psi$, that there exists $x_{\psi} \in W$ with $\psi\left(x_{\psi}\right) \leq c$. Then there exists $x \in W$ such that $\psi(x) \leq c$ for all $\psi \in \Psi$.

Recall that a family of functions $\mathcal{F}$ is said to be concave if for every finite collection of functions $\psi_{1}, \ldots, \psi_{n} \in \mathcal{F}$ and every finite collection of positive scalars $\alpha_{1}, \ldots, \alpha_{n}$ such that $\sum_{i=1}^{n} \alpha_{i}=1$, there is a function $\phi \in \mathcal{F}$ such that

$$
\sum_{i=1}^{n} \alpha_{i} \psi_{i} \leq \phi
$$

In the theorem below we consider the weak* topology generated by $X(\mu)_{[q]}$ on $\mathrm{M}\left(X(\mu)_{[q]}, Y(\mu)\right)$; it is a Hausdorff locally convex topology. If a subset $M \subseteq \mathrm{M}\left(X(\mu)_{[q]}, Y(\mu)\right)$ is compact with respect to the weak* generalized topology (wgt* for short) we say that $M$ is wgt*-compact. This notion is a straightforward generalization of the definition of compact sets with respect to the weak* topology by changing this topology by the weak* generalized topology. Note that if $Y(\mu)=L^{1}(\mu)$, then $\mathrm{M}\left(X(\mu)_{[q]}, Y(\mu)\right)$ coincides with the Köthe dual of $X(\mu)_{[q]}$ that is the dual of this space whenever $X(\mu)$ is $q$-compact and $\sigma$-order continuous (see [14] for more information about generalized duality on function spaces). We say that a finite family of non negative scalars $\alpha_{1}, \ldots, \alpha_{n} \in \mathbb{R}$ is convex if $\sum_{i=1}^{n} \alpha_{i}=$ 1. 
Theorem 1 Let $X(\mu)$ and $Y(\mu)$ be Banach function spaces and let $M \subseteq \mathrm{M}\left(X(\mu)_{[q]}, Y(\mu)\right)$ be a convex and wgt*-compact set of positive functions. Let $E$ be a Banach space, let $T: X(\mu) \rightarrow E$ be a linear continuous map and let $S \subseteq E^{\prime}$. Given a positive functional $y_{0}^{\prime} \in Y(\mu)^{\prime}$ and a constant $k>0$, the following assertions are equivalent:

(a) For all finite collections $f_{1}, \ldots, f_{n} \in X(\mu), x_{1}^{\prime}, \ldots, x_{n}^{\prime} \in S$ and every couple of convex families of non negative scalars $\alpha_{1}, \ldots, \alpha_{n}$ and $\beta_{1}, \ldots, \beta_{n}$,

$$
\sum_{i=1}^{n} \alpha_{i}^{\frac{1}{q}} \beta_{i}^{\frac{1}{q^{\prime}}}\left|\left\langle T f_{i}, x_{i}^{\prime}\right\rangle\right| \leq k \sup _{g \in M}\left(\sum_{i=1}^{n} \alpha_{i}\left\langle\left|f_{i}\right|^{q} g, y_{0}^{\prime}\right\rangle\right)^{\frac{1}{q}}\left(\sum_{i=1}^{n} \beta_{i}\left\|x_{i}^{\prime}\right\|^{q^{\prime}}\right)^{\frac{1}{q^{\prime}}}
$$

(b) There exists $g_{0} \in M$ such that

$$
\sup _{x^{\prime} \in S}\left|\left\langle T f, \frac{x^{\prime}}{\left\|x^{\prime}\right\|}\right\rangle\right| \leq k\left(\left\langle|f|^{q} g_{0}, y_{0}^{\prime}\right\rangle\right)^{\frac{1}{q}} \text {, for all } f \in X(\mu) .
$$

Proof:

(a) $\Rightarrow$ (b) First, fix $f_{1}, \ldots, f_{n} \in X(\mu), x_{1}^{\prime}, \ldots, x_{n}^{\prime} \in S$ and two convex families of scalars $\alpha_{1}, \ldots, \alpha_{n}$ and $\beta_{1}, \ldots, \beta_{n}$. Observe that using (1) and Young's Inequality which assure us: let a,b are nonnegative real numbers and, let $q$ and $q$ ' are positive real numbers such that $1 / q+1 / q^{\prime}=1$, then we have

$$
a b \leq \frac{a^{q}}{q}+\frac{b^{q^{\prime}}}{q^{\prime}}
$$

so, we obtain that

$$
\begin{aligned}
\sum_{i=1}^{n} \alpha_{i}^{1 / q} \beta_{i}^{1 / q^{\prime}}\left|\left\langle T f_{i}, x_{i}^{\prime}\right\rangle\right| & \leq k \sup _{g \in M}\left(\sum_{i=1}^{n} \alpha_{i}\left\langle\left|f_{i}\right|^{q} g, y_{0}^{\prime}\right\rangle\right)^{\frac{1}{q}}\left(\sum_{i=1}^{n} \beta_{i}\left\|x_{i}^{\prime}\right\|^{q^{\prime}}\right)^{1 / q^{\prime}} \\
& \leq \frac{k}{q} \sup _{g \in M}\left(\sum_{i=1}^{n} \alpha_{i}\left\langle\left|f_{i}\right|^{q} g, y_{0}^{\prime}\right\rangle\right)+\frac{k}{q^{\prime}} \sum_{i=1}^{n} \beta_{i}\left\|x_{i}^{\prime}\right\|^{q^{\prime}}(3)
\end{aligned}
$$

Let us define the function $\phi: M \rightarrow \mathbb{R}$ (depending on the functions $f_{1}, \ldots, f_{n} \in X(\mu)$, the functionals $x_{1}^{\prime}, \ldots, x_{n}^{\prime} \in S$ and the 
couple of families of non negative scalars $\alpha_{1}, \ldots, \alpha_{n}$ and $\beta_{1}, \ldots, \beta_{n}$ ) by

$$
\phi(g):=\sum_{i=1}^{n} \alpha_{i}^{\frac{1}{q}} \beta_{i}^{\frac{1}{q^{\prime}}}\left|\left\langle T f_{i}, x_{i}^{\prime}\right\rangle\right|-\frac{k}{q} \sum_{i=1}^{n} \alpha_{i}\left\langle\left|f_{i}\right|^{q} g, y_{0}^{\prime}\right\rangle-\frac{k}{q^{\prime}} \sum_{i=1}^{n} \beta_{i}\left\|x_{i}^{\prime}\right\|^{q^{\prime}}
$$

where $g \in M$.

A direct computation shows that the family $\mathcal{F}$, consisting of all such functions (for different sets of functions in $X(\mu)$, functionals in $S$ and couple of convex families of non negative scalars) is a concave family of convex functions. Moreover, $M$ is a convex subset of $\mathrm{M}\left(X(\mu)_{[q]}, Y(\mu)\right)$ that is compact with respect to the weak* topology generated by $X(\mu)_{[q]}$ on $\mathrm{M}\left(X(\mu)_{[q]}, Y(\mu)\right)$, and all the functions belonging to the family are wgt*-continuous.

Using (3), and taking into account the wgt*-compactness of $M$ we obtain that there is an element $g \in M$ such that $\sum_{i=1}^{n} \alpha_{i}\left\langle\left|f_{i}\right|^{q}(\cdot), y_{0}^{\prime}\right\rangle$ attains its maximum, and then the function $g$ satisfies that $\phi(g) \leq$ 0 . Since this happens for every function $\phi$ defined as above, Lemma 3 gives that there exists $g_{0} \in M$ such that $\phi\left(g_{0}\right) \leq 0$ for all $\phi \in \mathcal{F}$. In particular, for $f \in X(\mu)$ and $x^{\prime} \in S$, if $\left\langle|f|{ }^{q} g_{0}, y_{0}^{\prime}\right\rangle \neq 0$ take the function $\psi \in \mathcal{F}$ associated with the functional $x^{\prime} \in S$ and the function $\gamma f \in X(\mu)$ where $\gamma=\| x^{\prime}||^{q^{\prime} / q}\left(\left\langle|f|^{q} g, y_{0}^{\prime}\right\rangle\right)^{-1 / q}$ (together with $\alpha=1=\beta$ ). The linearity of $T$ gives

$$
\psi\left(g_{0}\right)=\gamma\left|\left\langle T f, x^{\prime}\right\rangle\right|-\frac{k}{q} \gamma^{q}\left\langle|f|^{q} g_{0}, y_{0}^{\prime}\right\rangle-\frac{k}{q^{\prime}}\left\|x^{\prime}\right\|^{q^{\prime}} \leq 0 .
$$

Hence,

$$
\left|\left\langle T f, x^{\prime}\right\rangle\right| \leq \frac{k}{q} \gamma^{q-1}\left\langle|f|^{q} g_{0}, y_{0}^{\prime}\right\rangle+\frac{k}{\gamma q^{\prime}}\left\|x^{\prime}\right\|^{q^{\prime}}=k\left(\left\langle|f|^{q} g_{0}, y_{0}^{\prime}\right\rangle\right)^{\frac{1}{q}}\left\|x^{\prime}\right\| .
$$

Now suppose that $\left\langle|f|^{q} g_{0}, y_{0}^{\prime}\right\rangle=0$. Note that the definition of the function $\phi$ given in (4) for the case $f \in X(\mu), x^{\prime} \in S$ and $\alpha=1=\beta$, and the property of $g_{0}$ give

$$
\left|\left\langle T f, x^{\prime}\right\rangle\right| \leq \frac{k}{q^{\prime}}\left\|x^{\prime}\right\|^{q^{\prime}}
$$

Since this inequality may happen for each $f \in X(\mu)$, we have that $\left|\left\langle T f, x^{\prime}\right\rangle\right|=0$, and then the inequality (5) is also fulfilled in this case. Therefore, we obtain (b). 
(b) $\Rightarrow$ (a) First note that the inequality in (b) is equivalent to $\left|\left\langle T f, x^{\prime}\right\rangle\right| \leq k\left(\left\langle|f|^{q} g_{0}, y_{0}^{\prime}\right\rangle\right)^{\frac{1}{q}}\left\|x^{\prime}\right\|$, for all $f \in X(\mu)$ and $x^{\prime} \in S$.

Thus, Hölder's Inequality provides the following inequalities, that prove (a).

$$
\begin{aligned}
\sum_{i=1}^{n} \alpha_{i}^{\frac{1}{q}} \beta_{i}^{\frac{1}{q^{\prime}}}\left|\left\langle T f_{i}, x_{i}\right\rangle\right| & \leq k \sum_{i=1}^{n}\left(\alpha_{i}\left\langle\left|f_{i}\right|^{q} g_{0}, y_{0}^{\prime}\right\rangle\right)^{\frac{1}{q}} \beta_{i}^{\frac{1}{q^{\prime}}}\left\|x_{i}^{\prime}\right\| \\
& \leq k\left(\sum_{i=1}^{n} \alpha_{i}\left\langle\left|f_{i}\right|^{q} g_{0}, y_{0}^{\prime}\right\rangle\right)^{\frac{1}{q}}\left(\sum_{i=1}^{n} \beta_{i}\left\|x_{i}^{\prime}\right\|^{q^{\prime}}\right)^{\frac{1}{q^{\prime}}} \\
& \leq k \sup _{g \in M}\left(\sum_{i=1}^{n} \alpha_{i}\left\langle\left|f_{i}\right|^{q} g, y_{0}^{\prime}\right\rangle\right)^{\frac{1}{q}}\left(\sum_{i=1}^{n} \beta_{i}\left\|x_{i}^{\prime}\right\|^{q^{\prime}}\right)^{\frac{1}{q^{\prime}}}
\end{aligned}
$$

Remark 1 Note that the compactness requirement for the set $M$ in Theorem 1 can be weakened. In fact, what is needed is the set to be compact for a topology with respect to which all the functions in the family $\mathcal{F}$ are continuous. But this topology is the one with respect to which all the functions $y_{0}^{\prime} \circ h$ are continuous - where $h \in$ $\mathrm{M}\left(X(\mu)_{[q]}, Y(\mu)\right)$-, that is clearly weaker than the wgt* topology. However, depending on the function $y_{0}^{\prime}$ this topology can even be not Hausdorff, since it may happen that the elements of $X(\mu)_{[q]}$ are not enough to separate the points of the space $\left\{y_{0}^{\prime} \circ h: h \in\right.$ $\left.\mathrm{M}\left(X(\mu)_{[q]}, Y(\mu)\right)\right\}$, so we prefer to use the stronger condition that appears in the statement of Theorem 1.

Remark 2 Given $0 \neq x^{\prime} \in E^{\prime}$, consider the set $S=\left\{x^{\prime}\right\}$ for Theorem 1. Take a $\sigma$-order continuous Banach function space $X(\mu)$ and $Y(\mu)=L^{1}(\mu)$, where $\mu$ is a positive measure. In this case it is known that $\mathrm{M}\left(X(\mu)_{[q]}, Y(\mu)\right)=\mathrm{M}\left(X(\mu)_{[q]}, L^{1}(\mu)\right)=\left(X(\mu)_{[q]}\right)^{\times}=$ $\left(X(\mu)_{[q]}\right)^{\prime}$, even in the case that $X(\mu)_{[q]}$ is just a quasi Banach space and not a Banach function space; this can be proved using the same argument that works for the case of Banach function spaces (see [13, p.29]). This proof holds by defining a measure associated with each functional, that is countably additive by the $\sigma$-order continuity of $X(\mu)_{[p]}$, and then use the Radon-Nikodým Theorem (see [17, Proposition 2.15(ii)]). Consider the set

$$
M=B_{\left(X(\mu)_{[q]}\right)^{\prime}}^{+}=\left\{\varphi \in B_{\left(X(\mu)_{[q]}\right)^{\prime}}: \varphi \geq 0\right\} .
$$


It is always weak* compact, also in the case that $X(\mu)_{[q]}$ is just a quasi Banach space (see the generalized Alaoglu Theorem in [19, Theorem 3.15]). Applying Theorem 1 with the positive functional $y_{0}^{\prime}=\chi_{\Omega} \in L^{\infty}(\mu)$, the inequality (2) can be rewritten as

$$
\begin{aligned}
\sum_{i=1}^{n} \alpha_{i}^{\frac{1}{q}} \beta_{i}^{\frac{1}{q^{\prime}}}\left|\left\langle T f_{i}, x^{\prime}\right\rangle\right| & \leq k \sup _{\varphi \in B_{(X(\mu)}^{+}[q]^{\prime}}\left(\int_{\Omega}\left(\sum_{i=1}^{n} \alpha_{i}\left|f_{i}\right|^{q}\right) \varphi d \mu\right)^{\frac{1}{q}}\left(\sum_{i=1}^{n} \beta_{i}\left\|x^{\prime}\right\|^{q^{\prime}}\right)^{\frac{1}{q^{\prime}}} \\
& \leq k \sup _{\varphi \in B_{(X(\mu)}^{+}[q]^{\prime}}\left(\sum_{i=1}^{n} \alpha_{i} \int_{\Omega}\left|f_{i}\right|^{q} \varphi d \mu\right)^{\frac{1}{q}}\left\|x^{\prime}\right\|,
\end{aligned}
$$

for all couple of convex families $\alpha_{1}, \ldots, \alpha_{n}$ and $\beta_{1}, \ldots, \beta_{n}$, since there is only one element in $S$. Taking into account that $\sum_{i=1}^{n}\left(\beta_{i}^{1 / q^{\prime}}\right)^{q^{\prime}}=$ 1, i.e. $\left(\beta_{i}^{1 / q^{\prime}}\right)_{i=1}^{n} \in B_{\ell^{\prime}}$, we obtain by duality for each fixed couple of families of functions $f_{1}, \ldots, f_{n}$ and scalars $\alpha_{1}, \ldots, \alpha_{n}$,

$$
\left(\sum_{i=1}^{n} \alpha_{i}\left|\left\langle T f_{i}, x^{\prime}\right\rangle\right|^{q}\right)^{\frac{1}{q}} \leq k \sup _{\left.\varphi \in B_{(X(\mu)}^{+}[q]\right]^{\prime}}\left(\sum_{i=1}^{n} \alpha_{i} \int_{\Omega}\left|f_{i}\right|^{q} \varphi d \mu\right)^{\frac{1}{q}}\left\|x^{\prime}\right\| .
$$

Since this must happen for every family of functions $f_{1}, \ldots, f_{n}$, we can eliminate the coefficients $\alpha_{i}$ in these inequalities and consider only the following kind of relations.

$$
\left(\sum_{i=1}^{n}\left|\left\langle T f_{i}, x^{\prime}\right\rangle\right|^{q}\right)^{\frac{1}{q}} \leq k \sup _{\left.\varphi \in B_{(X(\mu)}^{+}[q]\right]^{\prime}}\left(\sum_{i=1}^{n} \int_{\Omega}\left|f_{i}\right|^{q} \varphi d \mu\right)^{\frac{1}{q}}\left\|x^{\prime}\right\| .
$$

Therefore, for the case $E=X(\mu)$ and $T$ the identity map, Theorem 1 gives that the inequality

$$
\left(\sum_{i=1}^{n}\left|\left\langle f_{i}, x^{\prime}\right\rangle\right|^{q}\right)^{\frac{1}{q}} \leq k \sup _{\left.\varphi \in B_{(X(\mu)}^{+}[q]\right]^{\prime}}\left(\int_{\Omega}\left(\sum_{i=1}^{n}\left|f_{i}\right|^{q}\right) \varphi d \mu\right)^{\frac{1}{q}}\left\|x^{\prime}\right\|
$$

holds for every finite family $f_{1}, \ldots, f_{n} \in X(\mu)$ if and only if there is a function $0 \leq \varphi_{x^{\prime}} \in B_{\left(X(\mu)_{[q]}\right)^{\prime}}$ (depending on $x^{\prime}$ ) such that

$$
\left|\left\langle f, x^{\prime}\right\rangle\right| \leq k\left(\int_{\Omega}|f|^{q} \varphi_{x^{\prime}} d \mu\right)^{\frac{1}{q}}\left\|x^{\prime}\right\|, \quad \text { for all } f \in X(\mu) .
$$


Although Theorem 1 can be applied in many different situations, the case explained in Remark 2 for a single element $x^{\prime} \in X(\mu)^{\prime}$ allows us to obtain a result that characterizes the $q$-convexity of a space $X(\mu)$. Let us present first some technical results.

Lemma 4 (See $[\mathbf{1 3}$, p.55]) Let $E, F$ be Banach lattices and let $1 \leq q<\infty$. Let $T$ be a positive operator from $E$ into $F$. Then for every finite family $x_{1}, \ldots, x_{n} \in E$

$$
\left\|\left(\sum_{i=1}^{n}\left|T x_{i}\right|^{q}\right)^{\frac{1}{q}}\right\| \leq\|T\|\left\|\left(\sum_{i=1}^{n}\left|x_{i}\right|^{q}\right)^{\frac{1}{q}}\right\| .
$$

Using Lemma 4 we can easily prove that for every $x^{\prime} \in B_{X(\mu)^{\prime}}$ the functional $\left\langle\cdot, x^{\prime}\right\rangle$ is always $q$-concave and its concavity constant is less or equal than $\left\|x^{\prime}\right\|$.

Lemma 5 Let $X(\mu)$ be a Banach function space and let $x^{\prime} \in$ $X(\mu)^{\prime}$. Then for every family family $f_{1}, \ldots, f_{n} \in X(\mu)$

$$
\left(\sum_{i=1}^{n}\left|\left\langle f_{i}, x^{\prime}\right\rangle\right|^{q}\right)^{\frac{1}{q}} \leq\left\|x^{\prime}\right\|\left\|\left(\sum_{i=1}^{n}\left|f_{i}\right|^{q}\right)^{\frac{1}{q}}\right\| .
$$

Proof: $\quad$ Note that for all $f \in X(\mu)$ and $x^{\prime} \in X(\mu)^{\prime}$ we have that $\left|\left\langle f, x^{\prime}\right\rangle\right| \leq\left\langle|f|,\left|x^{\prime}\right|\right\rangle$. Hence, applying Lemma 4 we have that

$$
\left(\sum_{i=1}^{n}\left|\left\langle f_{i}, x^{\prime}\right\rangle\right|^{q}\right)^{\frac{1}{q}} \leq\left(\left.\sum_{i=1}^{n}\left\langle\left|f_{i}\right|,\left|x^{\prime}\right|\right\rangle\right|^{q}\right)^{\frac{1}{q}} \leq\left\|x^{\prime}\right\|\left\|\left(\sum_{i=1}^{n}\left|f_{i}\right|^{q}\right)^{\frac{1}{q}}\right\| .
$$

Theorem 2 Let $X(\mu)$ be a $\sigma$-order continuous Banach function space. Then the following assertions are equivalent:

(a) There is a constant $k>0$ such that for every norm one element $x^{\prime} \in X(\mu)^{\prime}$ and all finite collection $f_{1}, \ldots, f_{n} \in$ $X(\mu)$, the following inequality holds

$$
\left(\sum_{i=1}^{n}\left|\left\langle f_{i}, x^{\prime}\right\rangle\right|^{q}\right)^{\frac{1}{q}} \leq k \sup _{\varphi \in B_{(X(\mu)}^{+}[q]^{\prime}}\left(\int_{\Omega}\left(\sum_{i=1}^{n}\left|f_{i}\right|^{q}\right) \varphi d \mu\right)^{\frac{1}{q}}
$$


(b) There exists a constant $k>0$ satisfying that for each norm one element $x^{\prime} \in X(\mu)^{\prime}$ there is a function $\varphi_{x^{\prime}} \in B_{\left(X(\mu)_{[q]}\right)^{\prime}}^{+}$ such that

$$
\left|\left\langle f, x^{\prime}\right\rangle\right| \leq k\left(\int_{\Omega}|f|^{q} \varphi_{x^{\prime}} d \mu\right)^{\frac{1}{q}}, \text { for all } f \in X(\mu) .
$$

(c) The space $X(\mu)$ is q-convex.

Proof:

(a) $\Rightarrow$ (b) Just use Remark 2 .

(b) $\Rightarrow$ (c) Given $f_{1}, \ldots, f_{n} \in X(\mu)$ and using Hahn-Banach Theorem we can find a norm one element $x_{0}^{\prime} \in X(\mu)^{\prime}$ such that

$$
\left\|\left(\sum_{i=1}^{n}\left|f_{i}\right|^{q}\right)^{\frac{1}{q}}\right\|=\sup _{x^{\prime} \in B_{X(\mu)^{\prime}}}\left|\left\langle\left(\sum_{i=1}^{n}\left|f_{i}\right|^{q}\right)^{\frac{1}{q}}, x^{\prime}\right\rangle\right|=\left|\left\langle\left(\sum_{i=1}^{n}\left|f_{i}\right|^{q}\right)^{\frac{1}{q}}, x_{0}^{\prime}\right\rangle\right| .
$$

Hence, applying the inequality in (b) with $x_{0}^{\prime}$ there is $\varphi_{x_{0}^{\prime}} \in$ $B_{\left(X(\mu)_{[q]}\right)^{\prime}}^{+}$such that for the function $f=\left(\sum_{i=1}^{n}\left|f_{i}\right|^{q}\right)^{\frac{1}{q}} \in X(\mu)$ we obtain that

$$
\begin{aligned}
\left\|\left(\sum_{i=1}^{n}\left|f_{i}\right|^{q}\right)^{\frac{1}{q}}\right\| & \leq k\left(\int_{\Omega}\left(\sum_{i=1}^{n}\left|f_{i}\right|^{q}\right) \varphi_{x_{0}^{\prime}} d \mu\right)^{\frac{1}{q}}=k\left(\sum_{i=1}^{n} \int_{\Omega}\left|f_{i}\right|^{q} \varphi_{x_{0}^{\prime}} d \mu\right)^{\frac{1}{q}} \\
& \leq k\left(\sum_{i=1}^{n}\left\|\left|f_{i}\right|^{q}\right\|_{X(\mu)_{[q]}}\left\|\varphi_{x_{0}^{\prime}}\right\|_{\left(X(\mu)_{[q]}\right)^{\prime}}\right)^{\frac{1}{q}} \\
& \leq k\left\|\varphi_{x_{0}^{\prime}}\right\|_{\left(X(\mu)_{[q]}\right)^{\prime}}^{\frac{1}{q}}\left(\sum_{i=1}^{n}\left\|f_{i}\right\|_{X(\mu)}^{q}\right)^{\frac{1}{q}} \\
& \leq k\left(\sum_{i=1}^{n}\left\|f_{i}\right\|_{X(\mu)}^{q}\right)^{\frac{1}{q}} .
\end{aligned}
$$

Hence, $X(\mu)$ is $q$-convex.

(c) $\Rightarrow$ (a) Since $X(\mu)$ is a $q$-convex space then $X(\mu)_{[q]}$ is a Banach function space and the quasi-norm $\|\cdot\|_{X(\mu)_{[q]}}$ is equivalent to a norm, say $\|\cdot\|_{X(\mu)_{[q]}}$ (see Notation and preliminaries p.10). Let us take now $f_{1} \ldots, f_{n} \in X(\mu)$ and $x^{\prime}$ a norm one element in $X(\mu)^{\prime}$. Using Lemma 5 and the $\sigma$-order continuity of $X(\mu)_{[q]}$ — recall that if $X(\mu)$ 
is $\sigma$-order continuous then $X(\mu)_{[q]}$ is also $\sigma$-order continuous-, we obtain that

$$
\begin{aligned}
\left(\sum_{i=1}^{n}\left|\left\langle f_{i}, x^{\prime}\right\rangle\right|^{q}\right)^{\frac{1}{q}} & \leq\left\|x^{\prime}\right\|\left\|\left(\sum_{i=1}^{n}\left|f_{i}\right|^{q}\right)^{\frac{1}{q}}\right\|_{X(\mu)}=\left\|\left(\sum_{i=1}^{n}\left|f_{i}\right|^{q}\right)^{\frac{1}{q}}\right\|_{X(\mu)} \\
& =\left\|\sum_{i=1}^{n}\left|f_{i}\right|^{q}\right\|_{X(\mu)_{[q]}}^{\|^{\frac{1}{q}}} \leq k\left\|\sum_{i=1}^{n}\left|f_{i}\right|^{q}\right\|_{X(\mu)_{[q]}}^{\frac{1}{q}} \\
& =k \sup _{\left.\varphi \in B_{(X(\mu)}^{+}(q]\right]^{\prime}}\left(\int_{\Omega}\left(\sum_{i=1}^{n}\left|f_{i}\right|^{q}\right) \varphi d \mu\right)^{\frac{1}{q}} .
\end{aligned}
$$

\section{A topological characterization of the q-convexity.}

Given a Banach function space $X(\mu)$ and $1 \leq q<\infty$, we can consider the family

$$
\mathcal{T}=\left\{p_{\varphi}: 0 \leq \varphi \in B_{\left(X(\mu)_{[q]}\right)^{\prime}}\right\}
$$

of all the seminorms $p_{\varphi}: X(\mu) \rightarrow \mathbb{R}$ defined for each $0 \leq \varphi \in$ $B_{\left(X(\mu)_{[q]}\right)^{\prime}}$ as

$$
p_{\varphi}(f):=\left(\int_{\Omega}|f|^{q} \varphi d \mu\right)^{\frac{1}{q}}, \quad f \in X(\mu) .
$$

Note that for all $f_{1}, \ldots, f_{n} \in X(\mu)$ and $\varphi \in B_{\left(X(\mu)_{[q]}\right)^{\prime}}^{+}$the following equality holds

$$
\begin{aligned}
p_{\varphi}\left(\left(\sum_{i=1}^{n}\left|f_{i}\right|^{q}\right)^{\frac{1}{q}}\right) & =\left(\int_{\Omega}\left(\sum_{i=1}^{n}\left|f_{i}\right|^{q}\right) \varphi d \mu\right)^{\frac{1}{q}}=\left(\sum_{i=1}^{n} \int_{\Omega}\left|f_{i}\right|^{q} \varphi d \mu\right)^{\frac{1}{q}} \\
& =\left(\sum_{i=1}^{n}\left(p_{\varphi}\left(f_{i}\right)\right)^{q}\right)^{\frac{1}{q}}
\end{aligned}
$$

Consequently, $X(\mu)$ can be considered as a $q$-convex space if this property is defined with respect to all the seminorms $p_{\varphi} \in \mathcal{T}$, since $q$-convexity type inequalities - in fact, equalities - are satisfied for each seminorm of the family. 
Clearly we have that $p_{\varphi}(\cdot) \leq\|\cdot\|$ for all $p_{\varphi} \in \mathcal{T}$. Therefore, the topology $\tau_{q}$ generated by these seminorms is weaker than the topology of the norm, $\tau_{\|\cdot\| \cdot}$. On the other hand, if we consider in $X(\mu)$ the weak topology $\tau_{w}$ then Theorem 2 shows that if $X(\mu)$ is a $q$-convex space then the weak topology on $X(\mu), \tau_{w}$, is contained in $\tau_{q}$. The question that arises is: If the topology $\tau_{q}$ satisfies that $\tau_{w} \subseteq \tau_{q} \subseteq \tau_{\|\cdot\|}$, is the lattice $X(\mu) q$-convex? We will prove that this question has a positive answer. In fact, we will prove that if we have a family of $q$-concave seminorms with a similar property, this characterizes the $q$-convexity of the space. We start by defining the notions of $q$-convexity and weak $q$-convexity associated to a subset $S$ of $X(\mu)^{\prime}$. Note that both definitions are natural extensions of the concept of $q$-convexity, according to the results of the previous section.

Definition 17 Let $X(\mu)$ be a Banach function space and let $S \subseteq$ $B_{X(\mu)^{\prime}}$.

(a) We say that $X(\mu)$ is $(q, S)$-convex if there exists a constant $k>0$ satisfying that for all $f_{1}, \ldots, f_{n} \in X(\mu)$

$$
\left(\sum_{i=1}^{n}\left|\left\langle f_{i}, x^{\prime}\right\rangle\right|^{q}\right)^{\frac{1}{q}} \leq k \sup _{\varphi \in B_{(X(\mu)}^{+}[q]^{\prime}}\left(\int_{\Omega}\left(\sum_{i=1}^{n}\left|f_{i}\right|^{q}\right) \varphi d \mu\right)^{\frac{1}{q}}
$$

for all $x^{\prime} \in S$.

(b) We say that $X(\mu)$ is weakly $(q, S)$-convex if for each $x^{\prime} \in$ $S$ there exists a constant $k_{x^{\prime}}>0$ satisfying that for all $f_{1}, \ldots, f_{n} \in X(\mu)$

$$
\left(\sum_{i=1}^{n}\left|\left\langle f_{i}, x^{\prime}\right\rangle\right|^{q}\right)^{\frac{1}{q}} \leq k_{x^{\prime}} \sup _{\left.\varphi \in B_{(X(\mu)}^{+}[q]\right]^{\prime}}\left(\int_{\Omega}\left(\sum_{i=1}^{n}\left|f_{i}\right|^{q}\right) \varphi d \mu\right)^{\frac{1}{q}} .
$$

Note that the dual space of the $q$-th power of $X(\mu)$ can be trivial, and so there are $\sigma$-order continuous Banach function spaces -in fact, relevant spaces - that are not weakly $(q, S)$-convex; for instance, for every couple of real numbers $p, q$ such that $1 \leq p<q<$ $\infty$, and each non atomic measure $\mu,\left(\left(L^{p}(\mu)\right)_{[q]}\right)^{\prime}=\left(L^{\bar{p} / q}(\mu)\right)^{\prime}=$ $\{0\}$ (see for instance [7] or [10, Theorem 2.2]). Another direct consequence of Theorem 2 is that a $\sigma$-order continuous Banach 
function space $X(\mu)$ is $q$-convex, $1 \leq q<\infty$, if and only if it is $\left(q, B_{X^{\prime}(\mu)}\right)$-convex.

Obviously $(q, S)$-convexity implies weak $(q, S)$-convexity. In the following example we show that the converse is not true. In order to do this let us denote as usual by $c_{00}$ the set consisting of all sequences which components are eventually zero. Given $q>2$ we will prove that the space $\ell^{2}$ is weakly $\left(q, c_{00} \cap B_{\ell^{2}}\right)$-convex; however, it is not $\left(q, c_{00} \cap B_{\ell^{2}}\right)$-convex.

Example 1 Let us show that $\ell^{2}$ is weakly $\left(q, c_{00} \cap B_{\ell^{2}}\right)$-convex. Recall that $\left(\ell^{p}\right)^{\prime}=\ell^{\infty}$ for $0<p<1$ (see [7] or [10]). Since $\frac{2}{q}<1$ we can take the sequence

$$
\varphi_{0}=(1,1, \ldots) \in\left(\ell_{[q]}^{2}\right)^{\prime}=\left(\ell^{\frac{2}{q}}\right)^{\prime}=\ell^{\infty} .
$$

Then for all $f=\sum_{i=1}^{\infty} \lambda_{i} e_{i} \in \ell^{2} \subseteq \ell^{q}$ and for every $x^{\prime}=\sum_{j=1}^{N} \xi_{j} e_{j} \in$ $c_{00} \cap B_{\ell^{2}}$ - where $\left(e_{i}\right)_{i=1}^{\infty}$ is the canonical basis-we obtain

$$
\begin{aligned}
\left|\left\langle f, x^{\prime}\right\rangle\right| & =\left|\left\langle\sum_{i=1}^{\infty} \lambda_{i} e_{i}, \sum_{j=1}^{N} \xi_{j} e_{j}\right\rangle\right|=\left|\sum_{i=1}^{N} \lambda_{i} \xi_{i}\right| \\
& \leq\left(\sum_{i=1}^{\infty}\left|\lambda_{i}\right|^{q}\right)^{\frac{1}{q}}\left(\sum_{j=1}^{N}\left|\xi_{j}\right|^{q^{\prime}}\right)^{\frac{1}{q^{\prime}}}=\left(\int_{\Omega}|f|^{q} \varphi_{0} d \mu\right)^{\frac{1}{q}}\left\|x^{\prime}\right\|_{\ell^{q^{\prime}}} .
\end{aligned}
$$

Hence for all finite collection $f_{1}, \ldots, f_{n} \in \ell^{2}$ and for all $x^{\prime} \in$ $c_{00} \cap B_{\ell^{2}}$ we obtain

$$
\begin{aligned}
\left(\sum_{i=1}^{n}\left|\left\langle f_{i}, x^{\prime}\right\rangle\right|^{q}\right)^{\frac{1}{q}} & \leq\left\|x^{\prime}\right\|_{\ell^{q^{\prime}}}\left(\sum_{i=1}^{n} \int_{\Omega}\left|f_{i}\right|^{q} \varphi_{0} d \mu\right)^{\frac{1}{q}} \\
& =\left\|x^{\prime}\right\|_{\ell^{\prime}}\left(\int_{\Omega}\left(\sum_{i=1}^{n}\left|f_{i}\right|^{q}\right) \varphi_{0} d \mu\right)^{\frac{1}{q}} \\
& \leq\left\|x^{\prime}\right\|_{\ell^{q^{\prime}}} \sup _{\varphi \in B_{\ell \infty}^{+}}\left(\int_{\Omega}\left(\sum_{i=1}^{n}\left|f_{i}\right|^{q}\right) \varphi d \mu\right)^{\frac{1}{q}} \\
& =k_{x^{\prime}} \sup _{\varphi \in B_{\ell \infty}^{+}}\left(\int_{\Omega}\left(\sum_{i=1}^{n}\left|f_{i}\right|^{q}\right) \varphi d \mu\right)^{\frac{1}{q}} .
\end{aligned}
$$

So, $\ell^{2}$ is weakly $\left(q, c_{00} \cap B_{\ell^{2}}\right)$-convex for all $q>2$. However if we assume that $\ell^{2}$ is $\left(q, c_{00} \cap B_{\ell^{2}}\right)$-convex then we can find a constant $k>0$ such that for all finite collection $f_{1}, \ldots, f_{n} \in \ell^{2}$ and for all $x^{\prime} \in c_{00} \cap B_{\ell^{2}}$

$$
\left(\sum_{i=1}^{n}\left|\left\langle f_{i}, x^{\prime}\right\rangle\right|^{q}\right)^{\frac{1}{q}} \leq k \sup _{\varphi \in B_{\ell \infty}^{+}}\left(\int_{\Omega}\left(\sum_{i=1}^{n}\left|f_{i}\right|^{q}\right) \varphi d \mu\right)^{\frac{1}{q}} .
$$

Since $2<q<\infty$ then $-3 / 2<1 / q-3 / 2<-1$. So there is a real number $\alpha$ such that

$$
-\frac{3}{2}<\alpha<\frac{1}{q}-\frac{3}{2}=-\frac{1}{2}-\frac{1}{q^{\prime}} .
$$


Let us consider $x^{\prime}=N^{\alpha} \sum_{i=1}^{N} i^{1 / q^{\prime}} e_{i} \in \ell^{2}$ and $f=\sum_{i=1}^{N} i^{1 / q} e_{i} \in \ell^{2}$. Using the second inequality in (10) we have that

$$
\left\|x^{\prime}\right\|_{\ell^{2}}=N^{\alpha}\left(\sum_{i=1}^{N} i^{\frac{2}{q^{\prime}}}\right)^{\frac{1}{2}} \leq N^{\alpha}\left(N \cdot N^{\frac{2}{q^{\prime}}}\right)^{\frac{1}{2}}=N^{\alpha+\frac{1}{2}+\frac{1}{q^{\prime}}} \leq 1 .
$$

So, $x^{\prime} \in c_{00} \cap B_{\ell^{2}}$. On the other hand

$$
\left|\left\langle f, x^{\prime}\right\rangle\right|=\left|N^{\alpha} \sum_{i=1}^{N} i^{\frac{1}{q}+\frac{1}{q^{\prime}}}\right|=\left|N^{\alpha} \sum_{i=1}^{N} i\right|=N^{\alpha} \frac{N(N+1)}{2} \approx N^{\alpha+2}
$$

and

$$
\sup _{\varphi \in B_{\ell \infty}^{+}}\left(\int_{\Omega}|f|^{q} \varphi d \mu\right)^{\frac{1}{q}}=\left\||f|^{q}\right\|_{\ell^{1}}^{\frac{1}{q}}=\left(\sum_{i=1}^{n} i\right)^{\frac{1}{q}}=\left(\frac{N(N+1)}{2}\right)^{\frac{1}{q}} \approx N^{\frac{2}{q}}
$$

This means by (9) that

$$
\frac{N^{\alpha+2}}{N^{\frac{2}{q}}}=N^{\alpha+2-\frac{2}{q}} \leq k .
$$

But this is imposible regarding the first inequality in (10) and for any $q>2$.

Hence $\ell^{2}$ is not $\left(q, c_{00} \cap B_{\ell^{2}}\right)$-convex.

Theorem 3 Let $X(\mu)$ be a $\sigma$-order continuous Banach function space. Then, $X(\mu)$ is $q$-convex if and only if $X(\mu)$ is weakly $\left(q, B_{X(\mu)^{\prime}}\right)$ convex.

Proof: $\quad$ Let us assume first that $X(\mu)$ is $q$-convex. By Theorem 2 there exists a constant $k>0$ satisfying that for all $x^{\prime} \in B_{X(\mu)^{\prime}}$ we can find $0 \leq \varphi_{x^{\prime}} \in B_{\left(X(\mu)_{[q]}\right)^{\prime}}$ such that

$$
\left|\left\langle f, x^{\prime}\right\rangle\right| \leq k\left(\int_{\Omega}|f|^{q} \varphi_{x^{\prime}} d \mu\right)^{\frac{1}{q}}\left\|x^{\prime}\right\|, \quad f \in X(\mu) .
$$

If we consider a finite collection of $f_{1}, \ldots, f_{n} \in X(\mu)$ and $x^{\prime} \in$ $X(\mu)^{\prime}$, then

$$
\left|\left\langle f_{i}, x^{\prime}\right\rangle\right|^{q} \leq k^{q}\left(\int_{\Omega}\left|f_{i}\right|^{q} \varphi_{x^{\prime}} d \mu\right)\left\|x^{\prime}\right\|^{q}, \quad \text { for all } 1 \leq i \leq n .
$$


Hence

$$
\begin{aligned}
\left(\sum_{i=1}^{n}\left|\left\langle f_{i}, x^{\prime}\right\rangle\right|^{q}\right)^{\frac{1}{q}} & \leq k\left\|x^{\prime}\right\|\left(\int_{\Omega}\left(\sum_{i=1}^{n}\left|f_{i}\right|^{q}\right) \varphi_{x^{\prime}} d \mu\right)^{\frac{1}{q}} \\
& \leq k\left\|x^{\prime}\right\| \sup _{\varphi \in B_{(X(\mu)}^{+}(q]^{\prime}}\left(\int_{\Omega}\left(\sum_{i=1}^{n}\left|f_{i}\right|^{q}\right) \varphi d \mu\right)^{\frac{1}{q}}
\end{aligned}
$$

Thus $X(\mu)$ is weakly $\left(q, B_{X(\mu)^{\prime}}\right)$-convex. For the converse suppose that $X(\mu)$ is weakly $\left(q, B_{X(\mu)^{\prime}}\right)$-convex. Given a finite collection $f_{1}, \ldots, f_{n} \in X(\mu)$ let us define the bounded linear map $T_{f_{1}, \ldots, f_{n}}$ : $X(\mu)^{\prime} \rightarrow \ell^{q}$ by

$$
T_{f_{1}, \ldots, f_{n}}\left(x^{\prime}\right):=\left(\left\langle f_{i}, x^{\prime}\right\rangle\right)_{i=1}^{n}, \quad \text { for each } x^{\prime} \in X(\mu)^{\prime} .
$$

Let us consider now the family, which are contained in $L\left(X(\mu)^{\prime}, \ell^{q}\right)$ :

$\mathcal{A}=\left\{T_{f_{1}, \ldots, f_{n}} \mid f_{1} \ldots, f_{n} \in X(\mu), \sup _{\varphi \in B_{(X(\mu)}^{+}[q]^{\prime}}\left(\int_{\Omega}\left(\sum_{i=1}^{n}\left|f_{i}\right|^{q}\right) \varphi d \mu\right)^{\frac{1}{q}} \leq 1\right\}$

Clearly $\mathcal{A}$ is not an empty set. Since $X(\mu)$ is weakly $\left(q, B_{X(\mu)^{\prime}}\right)$ convex given $x^{\prime} \in X(\mu)^{\prime}$ there exists a constant $k_{\left(x^{\prime} /\left\|x^{\prime}\right\|\right)}>0$ such that

$$
\left\|T_{f_{1}, \ldots, f_{n}}\left(x^{\prime}\right)\right\| \leq k_{\left(x^{\prime} /\left\|x^{\prime}\right\|\right)}\left\|x^{\prime}\right\|, \quad \text { for all } T_{f_{1}, \ldots, f_{n}} \in \mathcal{A} .
$$

Using Banach-Steinhaus Theorem, we can find a constant $k>0$ such that

$$
\left\|T_{f_{1}, \ldots, f_{n}}\right\| \leq k, \text { for all } \quad T_{f_{1}, \ldots, f_{n}} \in \mathcal{A}
$$

Hence,

$$
\left(\sum_{i=1}^{n}\left|\left\langle f_{i}, x^{\prime}\right\rangle\right|^{q}\right)^{\frac{1}{q}}=\left\|T_{f_{1}, \ldots, f_{n}}\left(x^{\prime}\right)\right\| \leq k \sup _{\varphi \in B_{(X(\mu)}^{+}[q]^{\prime}}\left(\int_{\Omega}\left(\sum_{i=1}^{n}\left|f_{i}\right|^{q}\right) \varphi d \mu\right)^{\frac{1}{q}}
$$

for all $f_{1}, \ldots, f_{n} \in X(\mu)$ and each element $x^{\prime} \in B_{X(\mu)^{\prime}}$. But this means that the Banach function space $X(\mu)$ is $q$-convex, as a consequence of Theorem 2 .

In Remark 2 we have shown that weak $(q, S)$-convexity does not imply $(q, S)$-convexity. The space $\ell^{2}$ is 2 -convex but it is not $q$ convex for any $q>2$. However we have seen in Example 1 that it is weakly $\left(q, c_{00} \cap B_{\ell^{2}}\right)$-convex for all $q>2$, even though $c_{00}$ is a dense 
subset of $\ell^{2}$. In the following result we provide a condition to have the equivalence between weak $(q, S)$-convexity and $(q, S)$-convexity; the key is given by the fact that the Banach-Steinhaus Theorem can only be applied whenever the space where the operator is defined is complete.

Theorem 4 Let $X(\mu)$ be a $\sigma$-order continuous Banach function space and let $S \subseteq B_{X(\mu)^{\prime}}$ such that $\operatorname{span}(S)$ is closed in $X(\mu)^{\prime}$. Then, $X(\mu)$ is $(q, S)$-convex if and only if $X(\mu)$ is weakly $(q, S)$ convex.

Proof: $\quad$ Since $(q, S)$-convexity always implies weak $(q, S)$-convexity let us assume that $X(\mu)$ is weakly $(q, S)$-convex. Since $\operatorname{span}(S)$ is closed in the Banach space $X(\mu)^{\prime}$, then $\operatorname{span}(S)$ is complete; therefore, the proof is similar to the proof of Theorem 3 just changing $X(\mu)^{\prime}$ by $\operatorname{span}(S)$ in the definition of the linear maps $T_{f_{1}, \ldots, f_{n}}-$ i.e. the same argument but considering the family $\mathcal{A}$ included in $L\left(\operatorname{span}(S), \ell^{q}\right)$, see the definition given in $(11)-$.

Corollary 2 Let $X(\mu)$ be a $\sigma$-order continuous Banach function space. The following assertions are equivalent:

(a) The space $X(\mu)$ is $q$-convex.

(b) The inclusions $\tau_{w} \subseteq \tau_{q} \subseteq \tau_{\|\cdot\|}$ hold.

Proof:

(a) $\Rightarrow$ (b) Is just a consequence of Theorem 2. This result clearly provides the inclusion $\tau_{w} \subseteq \tau_{q}$, and the other inclusion, $\tau_{q} \subseteq \tau_{\|\cdot\|}$, always holds.

(b) $\Rightarrow$ (a) If the first inclusion in (b) hold, then for each norm one element $x^{\prime} \in X(\mu)^{\prime}$ there exists a constant $k_{x^{\prime}}>0$ satisfying that there is function $\varphi_{x^{\prime}} \in B_{\left(X(\mu)_{[q]}\right)^{\prime}}^{+}$such that

$$
\left|\left\langle f, x^{\prime}\right\rangle\right| \leq k_{x^{\prime}}\left(\int_{\Omega}|f|^{q} \varphi_{x^{\prime}} d \mu\right)^{\frac{1}{q}}, \quad \text { for all } f \in X(\mu),
$$

since the sum $\sum_{i=1}^{n}\left|\varphi_{i}\right|$ for functions $\varphi_{1}, \ldots, \varphi_{n} \in\left(X(\mu)_{[q]}\right)^{\prime}$ gives other function in this space. This clearly implies that $X(\mu)$ is weakly $\left(q, B_{X(\mu)^{\prime}}\right)$-convex. Thus, by Theorem $3, X(\mu)$ is $q$-convex.

In the previous result we have shown that the fact that the topology $\tau_{q}$ generated by the seminorms $p_{\varphi}$ satisfies the relations 
$\tau_{w} \subseteq \tau_{q} \subseteq \tau_{\|\cdot\|}$-we can just take functions $0 \leq \varphi \in B_{\left(X(\mu)_{[q]}\right)^{\prime}}$ characterizes when the space $X(\mu)$ is $q$-convex. On the other hand the seminorms $p_{\varphi}$ are $q$-concave (see the equalities in (8)). A natural question now is the following: What happens if we have the inclusions $\tau_{w} \subseteq \tau_{\mathcal{P}} \subseteq \tau_{\|\cdot\|}$ where $\tau_{\mathcal{P}}$ is the topology generated by any family of q-concave seminorms? We will prove that if these inclusions hold then $X(\mu)$ is a $q$-convex space if and only if $\tau_{\mathcal{P}} \subseteq \tau_{q}$. Assume then that $p$ is a $q$-concave seminorm, i.e., for all $f_{1}, \ldots, f_{n} \in X(\mu)$

$$
\left(\sum_{i=1}^{n} p\left(f_{i}\right)^{q}\right)^{\frac{1}{q}} \leq p\left(\left(\sum_{i=1}^{n}\left|f_{i}\right|^{q}\right)^{\frac{1}{q}}\right) .
$$

Note that the $q$-sum of a finite set of $q$-concave seminorms gives other $q$-concave seminorm. This means that we can restrict our attention to the set of fundamental seminorms of $\tau_{\mathcal{P}}$ regarding the inequalities that proves the coincidence of the topologies involved in the following characterization.

Corollary 3 Let $X(\mu)$ be a $\sigma$-order continuous Banach function space and $1 \leq q<\infty$. Assume that $\tau_{w} \subseteq \tau_{\mathcal{P}} \subseteq \tau_{\|\cdot\|}$, where $\tau_{\mathcal{P}}$ is a particular family of $q$-concave seminorms. Then the following assertions are equivalent:

(a) The space $X(\mu)$ is $q$-convex.

(b) The inclusion $\tau_{\mathcal{P}} \subseteq \tau_{q}$ holds.

Proof:

(a) $\Rightarrow$ (b) As in the proof of Theorem 1, we use a separation argument based on Lemma 3, so we only give a sketch of the proof. Since $\tau_{\mathcal{P}} \subseteq \tau_{\|\cdot\|}$ then for any seminorm $p$ belonging to $\tau_{\mathcal{P}}$ there is a constant $k_{p}>0$ satisfying that $p(f) \leq k_{p}\|f\|$ for every $f \in X(\mu)$. Since $X(\mu)$ is a $q$-convex space then $X(\mu)_{[q]}$ is a Banach function space and the quasi-norm $\|\cdot\|_{X(\mu)_{[q]}}$ is equivalent to a norm, say 
$\|\cdot\|_{X(\mu)_{[q]}}$. Using the $q$-concavity of the seminorms we obtain

$$
\begin{aligned}
\left(\sum_{i=1}^{n} p\left(f_{i}\right)^{q}\right)^{\frac{1}{q}} & \leq p\left(\left(\sum_{i=1}^{n}\left|f_{i}\right|^{q}\right)^{\frac{1}{q}} \leq k_{p}\left\|\left(\sum_{i=1}^{n}\left|f_{i}\right|^{q}\right)^{\frac{1}{q}}\right\|\right. \\
& =k_{p}\left\|\sum_{i=1}^{n}\left|f_{i}\right|^{q}\right\|_{X(\mu)_{[q]}}^{\frac{1}{q}} \leq c_{p}\left\|\sum_{i=1}^{n}\left|f_{i}\right|^{q}\right\|_{X(\mu)_{[q]}}^{\frac{1}{q}} \\
& =c_{p} \sup _{\varphi \in B_{(X(\mu)}^{+}\left([q]^{\prime}\right.}\left(\int_{\Omega}\left(\sum_{i=1}^{n}\left|f_{i}\right|^{q}\right) \varphi d \mu\right)^{\frac{1}{q}},
\end{aligned}
$$

for every finite family $f_{1}, \ldots, f_{n} \in X(\mu)$. For such a finite family, let us consider the function $\phi: B_{\left(X(\mu)_{[q]}\right)^{\prime}}^{+} \rightarrow \mathbb{R}$ (depending on the functions $\left.f_{1}, \ldots, f_{n} \in X(\mu)\right)$ given by

$$
\phi(\varphi):=\sum_{i=1}^{n} p\left(f_{i}\right)^{q}-c_{p}^{q} \int_{\Omega}\left(\sum_{i=1}^{n}\left|f_{i}\right|^{q}\right) \varphi d \mu .
$$

It is easy to check that the family $\mathcal{F}$ consisting of all such functions - for different finite sets of functions in $X(\mu)$ - is a concave family of convex functions. Moreover, $B_{\left(X(\mu)_{[q]}\right)^{\prime}}^{+}$is compact with respect to the weak* topology, and all the functions belonging to the family are weak* continuous. Hence we obtain that for every function $\phi \in \mathcal{F}$ we can find a function $\varphi \in B_{\left(X(\mu)_{[q]}\right)^{\prime}}^{+}$such that $\phi(\varphi) \leq 0$. So applying Lemma 3 there exists a function $0 \leq \varphi_{0} \in B_{\left(X(\mu)_{[q]}\right)^{\prime}}$ such that $\phi\left(\varphi_{0}\right) \leq 0$ for all $\phi \in \mathcal{F}$. Taking the family consisting of a single function $f \in X(\mu)$ we have that

$$
p(f)^{q} \leq c_{p}^{q} \int_{\Omega}|f|^{q} \varphi_{0} d \mu .
$$

Since the function $\varphi_{0}$ is the same for every $f \in X(\mu)$, we obtain (b).

(b) $\Rightarrow$ (a) If the inclusion in (b) holds then just observe that $\tau_{w} \subseteq$ $\tau_{\mathcal{P}} \subseteq \tau_{q} \subseteq \tau_{\|\cdot\|}$. Hence the result is a direct consequence of Corollary 2 .

Remark 3 Note that the arguments that has been used for obtaining the topological characterization of the q-convexity of a Banach function space strongly depend on the fact that we are dealing with function spaces. Actually, the q-th power can only be defined in 
the case that the elements of the lattice are functions, since its is defined point wise. As far as we know, there are no similar constructions in the case of abstract Banach lattices that can generalize our arguments. Note that the q-convexification/concavification for an abstract Banach lattice given in [13, p.53] cannot be handle in the same way that in the case of functions, since we need a useful representation of the Köthe dual of the spaces involved. Also $\sigma$-order continuity (that coincides with order continuity for Banach function spaces) is necessary for obtaining the right representation of the dual spaces; however, this requirement is not needed for the result of A. Schep quoted in the Introduction ([21, Prop.1.2]), where a weaker condition is assumed. So we propose the following open question: Is it possible to obtain characterizations of q-convexity in the case of abstract Banach lattices like the ones that are given by Corollary 2 and Corollary 3 ?

However, notice that our description of the family of q-concave seminorms, that seems natural for the case of $L^{q}$ spaces, works also for the case of other well-known Banach function spaces in which the q-power do not appear explicitly: Lorentz spaces, Orlicz spaces and Calderón-Lozanovskii spaces ([6, 8,9]).

\section{Applications: A Factorization Theo- rem for $(q, S)$-convex spaces.}

Let $E$ be a Banach space and $X(\mu)$ be a $\sigma$-order continuous Banach function space. Given $1 \leq q<\infty$, if $X(\mu)$ is $q$-convex then a generalized version of Maurey-Rosenthal Theorem states that each $q$-concave operator from $X(\mu)$ into $E$ factorizes through $L^{q}(\mu)$ (see, e.g., $[2,3,4,15,17])$. We finish this work with a version of MaureyRosenthal Theorem for $(q, S)$-convex $\sigma$-order continuous Banach function spaces. Given $S \subseteq B_{X(\mu)^{\prime}}$ we define the $(q, S)$-concavity of an operator from a Banach function space $X(\mu)$ into a Banach space $E$ as the dual notion of $(q, S)$-convexity.

First of all, we remember to the reader two versions of the Maurey-Rosenthal's Theorem for comparing the new results obtained through the concept of $(q, S)$-convexity. The second one can be seen in [17]. In fact, what is traditionally known as the MaureyRosenthal Theorem is a result regarding factorizations through $L^{2}(\mu)$ for operators taking their values in $L^{1}(\mu)$, (see [23], Theorem 12.30). 
Theorem 5 (Maurey-Rosenthal) Let $1<q<\infty, T: X(\mu) \rightarrow$ $Y$ be an operator, $X(\mu)$ a q-convex $\sigma$-order continuous Banach function space and $Y$ a Banach space. Then the following assertions are equivalent:

- $T$ is q-concave operator.

- $T$ factorizes through $L^{q}(\mu)$ as follows:

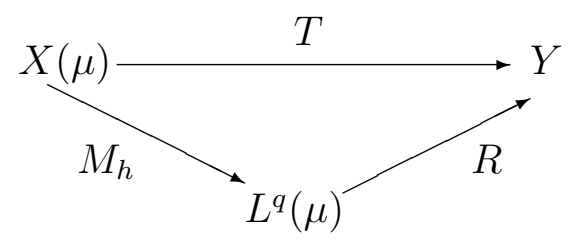

where $R$ is a continuous operator and $M_{h} \in M\left(X(\mu), L^{q}(\mu)\right)$ with $h$ measurable non-negative function.

Theorem 6 (Maurey-Rosenthal) Let $1 \leq q<\infty$ and let $X(\mu)$ be a $\sigma$-order continuous q-convex B.f.s. Consider a Banach space $E$ and a q-concave linear operator $T: X(\mu) \rightarrow E$. Then there exists $g \in L^{1}(\mu)$ satisfying:

$$
\sup _{\|f\| \leq 1}\left(\int_{\Omega}|f|^{q} g d \mu\right)^{1 / q} \leq M_{(q)}[T] \cdot M^{(q)}[X(\mu)]
$$

and

$$
\|T(f)\|_{E} \leq\left(\int_{\Omega}|f|^{q} g d \mu\right)^{1 / q}
$$

for every $f \in X(\mu)$. Under the above assumptions, $T$ factorizes as

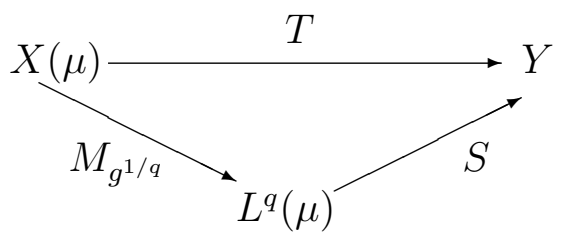

where $S$ is a continuous linear operator, $M_{g^{1 / q}}$ is the continuous operator of multiplication by $g^{1 / q}$ and $\left\|M_{g^{1 / q}}\right\| \cdot\|S\| \leq M_{(q)}[T]$. $M^{(q)}[X(\mu)]$.

Definition 18 A linear and continuous map $T$ from a Banach function space $X(\mu)$ into a Banach space $E$ is said to be $(q, S)$ concave, where $S \subseteq B_{X(\mu)^{\prime}}$, if there is a constant $k>0$ satisfying 
that for all $f_{1}, \ldots, f_{n} \in X(\mu)$

$$
\left(\sum_{i=1}^{n}\left\|T f_{i}\right\|^{q}\right)^{\frac{1}{q}} \leq k \sup _{x^{\prime} \in S}\left|\left\langle\left(\sum_{i=1}^{n}\left|f_{i}\right|^{q}\right)^{\frac{1}{q}}, x^{\prime}\right\rangle\right|
$$

Theorem 7 (Factorization Theorem) Let $E$ be a Banach space and let $X(\mu)$ be a $\sigma$-order continuous $(q, S)$-convex Banach function space, where $S \subseteq B_{X(\mu)^{\prime}}$. Given a $(q, S)$-concave operator $T: X(\mu) \rightarrow E$, there exist a constant $k>0$ and a function $0 \leq \varphi \in B_{\left(X(\mu)_{[p]}\right)^{\prime}}$ satisfying that for all $f \in X(\mu)$

$$
\|T f\| \leq k\|f\|_{L^{q}(\varphi d \mu)}
$$

or equivalently, $T$ can be factorized as

$$
X(\mu) \underset{M_{\varphi^{1 / q}} \quad R}{\longrightarrow} E .
$$

Proof: $\quad$ Since $X(\mu)$ is $(q, S)$-convex we have that there exists a constant $k_{1}>0$ satisfying that for each $f_{1}, \ldots, f_{n} \in X(\mu)$ and all $x^{\prime} \in S$,

$$
\left(\sum_{i=1}^{n}\left|\left\langle f_{i}, x^{\prime}\right\rangle\right|^{q}\right)^{\frac{1}{q}} \leq k_{1} \sup _{\left.\varphi \in B_{(X(\mu)}^{+}[q]\right]^{\prime}}\left(\int_{\Omega}\left(\sum_{i=1}^{n}\left|f_{i}\right|^{q}\right) \varphi d \mu\right)^{\frac{1}{q}} .
$$

In particular, taking the single function $f=\left(\sum_{i=1}^{n}\left|f_{i}\right|^{q}\right)^{\frac{1}{q}} \in X(\mu)$ we have that

$$
\left|\left\langle\left(\sum_{i=1}^{n}\left|f_{i}\right|^{q}\right)^{\frac{1}{q}}, x^{\prime}\right\rangle\right| \leq k_{1} \sup _{\varphi \in B_{(X(\mu)}^{+}[q]^{\prime}}\left(\int_{\Omega}\left(\sum_{i=1}^{n}\left|f_{i}\right|^{q}\right) \varphi d \mu\right)^{\frac{1}{q}} .
$$


Using the $(q, S)$-concavity we can find a constant $k_{2}>0$ such that

$$
\begin{aligned}
\left(\sum_{i=1}^{n}\left\|T f_{i}\right\|^{q}\right)^{\frac{1}{q}} & \leq k_{2} \sup _{x^{\prime} \in S}\left|\left\langle\left(\sum_{i=1}^{n}\left|f_{i}\right|^{q}\right)^{\frac{1}{q}}, x^{\prime}\right\rangle\right| \\
& \leq k_{1} k_{2} \sup _{x^{\prime} \in S} \sup _{\varphi \in B_{(X(\mu)}^{+}(q]^{\prime}}\left(\int_{\Omega}\left(\sum_{i=1}^{n}\left|f_{i}\right|^{q}\right) \varphi d \mu\right)^{\frac{1}{q}} \\
& =k \sup _{\varphi \in B_{(X(\mu)}^{+}(q]^{\prime}}\left(\int_{\Omega}\left(\sum_{i=1}^{n}\left|f_{i}\right|^{q}\right) \varphi d \mu\right)^{\frac{1}{q}} .
\end{aligned}
$$

An similar argument to the one that proves Corollary 3 gives a function $0 \leq \varphi_{0} \in B_{\left(X(\mu)_{[q]}\right)^{\prime}}$ such that the inequality

$$
\|T f\| \leq k\left(\int_{\Omega}|f|^{q} \varphi_{0} d \mu\right)^{\frac{1}{q}}
$$

holds for every $f \in X(\mu)$. This inequality provides the factorization of the operator. To see this, note that we can assume that $\varphi_{0}>0$. Since the range of the operator $M_{\varphi_{0}^{1 / q}}$ is dense in $L^{q}(\mu)$ (see the proof of Lemma 3.3 in [3]), then the function $R(g):=T\left(\varphi^{-1 / q} g\right)$ is well defined and continuous in the range of the operator $M_{\varphi_{0}^{1 / q}}$, so we can extend $R$ to $L^{q}(\mu)$. This finishes the proof.

\section{References}

[1] Calabuig, J.M, Delgado, O. and Sánchez Pérez, E.A., Generalized perfect spaces. Indag. Math. (N.S.), 19, 359-378 (2008).

[2] Defant, A., Variants of the Maurey-Rosenthal Theorem for Quasi Banach function spaces. Positivity 5, 153-175 (2001).

[3] Defant, A. and Sánchez Pérez, E.A., Maurey-Rosenthal factorization of positive operators and convexity. J. Math. Anal. Appl., 297, 771-790 (2004).

[4] Defant, A. and Sánchez Pérez, E.A., Domination of operators on function spaces. Math. Proc. Camb. Phil. Soc., 146, 57-66 (2009). 
[5] Diestel, J., Jarchow, H. and Tonge, A., Absolutely Summnig Operators, Cambridge Studies in Advanced Mathematics. 43. Cambridge University Press, Cambridge, 1995.

[6] Hao, C., Kamińska, A. and Tomczak-Jaegermann, N., Orlicz spaces with convexity or concavity constant one. J. Math. Anal. Appl., 320, 303-321 (2006).

[7] Kalton, N.J., Quasi-Banach spaces, In: Handbook of the Geometry of Banach Spaces, Volume 2, Eds. Johnson, W. B. and Lindenstrauss, J., 1099-1130. North-Holland, Amsterdam, 2003.

[8] Kamińska, A., Maligranda, L. and Persson, L.E., Convexity, concavity, type and cotype of Lorentz spaces. Indag. Math. (N.S.), 9, 367-382 (1998).

[9] Kamińska, A., Maligranda, L. and Persson, L.E., Indices, convexity and concavity of Calderón-Lozanovskii spaces. Math. Scand., 92, 141-160 (2003).

[10] Kalton, N.J., Peck, N.T. and Roberts, J.W., An F-space Sampler. Cambridge University Press (London Math. Soc. Lecture Notes Series 89), Cambridge, 1984.

[11] Krivine, J.L., Théorèmes de factorisation dans les espaces reticulés. Seminaire Maurey-Schwartz 1973-74, Exposés 2223, École Polytechnique, Paris, 1974.

[12] Lacey, H.E., The Isometric Theory of Classical Banach Spaces. Die Grundlagen der mathematischen Wissenschaften, Band 208. Springer, Berlin, 1974.

[13] Lindenstrauss, J. and Tzafriri, L., Classical Banach Spaces II. Springer, Berlin, 1996.

[14] Maligranda, L. and Persson, L.E., Generalized duality of some Banach function spaces. Indagationes Mathematicae 51(3), 323-338 (1989).

[15] Maurey, B., Théorèmes de factorisation pour les opérateurs linéaires à valeurs dans les spaces $L^{p}$. Astérisque 11 (1974).

[16] Meyer-Nieberg, P.M., Banach Lattices. Springer, Berlin, 1991. 
[17] S. Okada, W. J. Ricker and E. A. Sánchez-Pérez, Optimal domain and integral extension of operators acting in function spaces., Operator Theory: Advances and Applications, vol. 180, Birkhäuser Verlag, Basel, 2008.

[18] Rosenthal, H.P., On subspaces of $L^{p}$. Ann. of Math. 97, 344373 (1973).

[19] Rudin, W. Functional Analysis. McGraw Hill, New York, 1973.

[20] Sánchez Pérez, E.A. Asymptotic domination of operators on Köthe function spaces and convergence of sequences. Math. Nachr. 279 no. 15, 1709-1722 (2006).

[21] Schep, A.R. Minkowski's integral inequality for function norms. In: Operator Theory in Function Spaces and Banach Lattices. Operator Theory: Advances and Applications, vol. 75, Birkhäuser, Basel, 1995.

[22] Köthe, G., Topological Vector Spaces I ( 2nd printing, revised), Springer-Verlag, Berlin-Heidelberg-New York, 1983.

[23] Diestel, J., Jarchow, H. and Tonge, A., Absolutely Summing Operators. Cambridge Univ. Press, Cambridge, 1995. 